\title{
Impactos de la contaminación por basura marina en el ecosistema de manglar de la Ciénaga Grande de Santa Marta, Caribe colombiano
}

\section{Impacts of marine debris contamination in the mangrove ecosystem of the Ciénaga Grande de Santa Marta, Colombian Caribbean}

\author{
Ostin Garcés Ordóñez ${ }^{1 *}$ y Mónica Rocío Bayona Arenas ${ }^{2}$
}

\begin{abstract}
RESUMEN
La basura en el mar está generando impactos negativos en los ecosistemas marinos y comunidades humanas, razón por la cual se considera un problema relevante cuya solución ha sido incluida como meta dentro del Objetivo de desarrollo sostenible n. ${ }^{\circ}$ 14. El propósito de este estudio fue identificar y valorar los impactos negativos de la contaminación por basura marina en el ecosistema de manglar de la Ciénaga Grande de Santa Marta (CGSM), Caribe colombiano. Los impactos se identificaron mediante observación in situ (impactos observados), durante salidas de campo realizadas en febrero de 2018 y a partir de la revisión de información secundaria (impactos potenciales). La valoración de la importancia ambiental de los impactos observados se realizó tomando como base la metodología propuesta por Fernández (2010), que considera los criterios de intensidad, extensión, momento, reversibilidad, recuperabilidad, sinergia y acumulación. En total, se identificaron 10 observados en campo y 11 potenciales causados por la basura marina en los manglares de la CGSM. La presencia, la acumulación y la degradación de la basura marina generan efectos moderados, severos y críticos en el manglar de la CGSM. De estos se resaltan, por el peligro potencial, la acumulación de microplásticos en el ambiente, la limitación del establecimiento de propágulos y crecimiento de plántulas, y los cambios físicos en el suelo. Estos resultados servirán para que las autoridades ambientales puedan tomar acciones preventivas y reductivas de este tipo de contaminación y contribuir, significativamente, a la recuperación del manglar y sus servicios ambientales.
\end{abstract}

Palabras clave: Contaminación marina, impactos potenciales, microplásticos, residuos sólidos, valoración de impactos

1 Instituto de Investigaciones Marinas y Costeras “José Benito vives de Andréis”-INVEMAR. Calle 25 No. 2-55 playa Salguero, Santa Marta, Colombia. ostin.garces@invemar.org.co*

2 Ecopetrol S.A., Bogotá, Colombia. monicabayona85@gmail.com 


\section{ABSTRACT}

Marine debris generates negative impacts on marine ecosystems and human communities, which is why the solution to this relevant problem has been included in the Sustainable Development Goal No. 14. The aim of this study was to identify and assess the negative impacts of marine debris pollution on the mangrove ecosystem in the Ciénaga Grande de Santa Marta (CGSM), in the Colombian Caribbean. Impacts were identified through in situ observation (observed impacts) during field trips made in February 2018 and after reviewing the secondary information (potential impacts). Observed impacts were assessed based on the methodology proposed by Fernández-Vítora (2010), which takes into consideration the following criteria: intensity, extent, momentum, reversibility, recoverability, synergy and accumulation. In total, 10 impacts were identified in the field together with 11 potential impacts caused by marine litter in the CGSM mangroves. Presence, accumulation, and degradation of marine debris generate moderate, severe and critical impacts in the CGSM mangroves. Highlighted impacts due to their potential hazard to the ecosystem include microplastics accumulation, limitation of the propagules establishment, seedling growth, and physical changes in the soil. These results are useful for environmental authorities to take preventive measures and significant debris reduction actions and contribute to mangrove recovery and its environmental services.

Keywords: Marine pollution, potential impacts, microplastics, solid waste, impact assessment

\section{INTRODUCCIÓN}

Se considera como basura marina cualquier material sólido persistente, manufacturado o procesado, y que ha sido descartado, vertido o eliminado en el ambiente marino y costero (UNEP \& GRID-Arendal, 2016). El 80\% de la basura marina proviene de actividades en tierra, por el inadecuado manejo de residuos domésticos, la agricultura, la construcción y el turismo costero; el restante $20 \%$ proviene de actividades en el mar, como las industrias pesquera y naviera, la acuicultura, la pesca recreativa, y el transporte marítimo (UNEP \& GRID-Arendal, 2016). El plástico representa entre el $42 \%$ y $96 \%$ de la basura marina. Este llega al ambiente marino y costero por diferentes actividades y medios, como los ríos, el viento y los vertimientos directos de residuos (Jambeck et al. 2015; Iñiguez et al. 2016; UNEP \& GRID-Arendal, 2016; Lebreton et al. 2017).

Una subcategoría de los plásticos son los microplásticos (partículas con tamaño $<5 \mathrm{~mm}$ de diámetro), que representan un problema mayor por su abundancia en los ecosistemas y su capacidad de introducir compuestos orgánicos tóxicos y metales pesados del ambiente a la red trófica, con lo cual generan efectos adversos en las especies que acumulan estos tóxicos (Wang et al. 2016; Weinstein et al. 2016; Antão et al. 2018; Alimba \& Faggio, 2019). Además, los microplásticos son vectores de microorganismos patógenos para peces y seres humanos, 
como las bacterias de Escherichia coli, Vibrio spp y Aeromonas spp (Foulon et al. 2016; Kirstein et al. 2016; Kovač et al. 2017). Aunque actualmente se ha avanzado en el conocimiento sobre la distribución y abundancia de la basura marina (ver mapa de distribución en https://litterbase.awi.de/litter), aún existen importantes vacíos en el conocimiento sobre los impactos y efectos de los microplásticos en los ecosistemas y en la salud humana (Horton et al. 2017; Troost et al. 2018; Rainieri \& Barranco, 2019; Novotna et al. 2019; Prata et al. 2019a). A pesar de los reconocidos impactos de la basura, y de los microplásticos en específico, hay pocos estudios disponibles a nivel global en los ecosistemas de manglar (Cordero \& Costa, 2010; Debrot et al. 2013; Mohamed \& Obbard, 2014; Naji et al. 2017; Li et al. 2018; Martin et al. 2019; Garcés-Ordóñez et al. 2019).

El manglar es un ecosistema muy productivo conformado por plantas con raíces fúlcreas y neumatóforos que, además de ayudar al intercambio gaseoso y estabilización de la planta en sustratos inestables, funcionan como trampas de basura proveniente de fuentes terrestres y marítimas (Ivar et al. 2014; Martin et al. 2019). En los manglares de Nueva Guinea (Smith, 2012), Venezuela (Debrot et al. 2013), Arabia Saudita (Martin et al. 2019), Singapore (Mohamed \& Obbard, 2014), Irán (Naji et al. 2017) y Colombia (Riascos et al. 2019; Gar-
cés-Ordóñez et al. 2019) se han encontrado altas cantidades de basura y microplásticos que afectan la calidad ambiental del ecosistema.

La acumulación de basura marina en los manglares colombianos está asociada al inadecuado manejo de residuos municipales, ya que las poblaciones costeras disponen inadecuadamente el $\sim 65 \%$ de sus residuos sólidos en botaderos a cielo abierto, quemándolos, enterrándolos en el suelo o arrojándolos a cuerpos de aguas naturales como los ríos y lagunas (Garcés-Ordóñez et al. 2017; Garcés-Ordóñez et al. 2019; Riascos et al. 2019). Este tipo de contaminación marina genera diferentes impactos negativos en los ecosistemas (deterioro de la calidad del hábitat, introducción de especies invasoras, mortalidad y morbilidad de especies), en la salud humana (lesiones inflamatorias, enfermedades neurodegenerativas, trastornos inmunes y cánceres) y en la economía (incremento en los costos en la limpieza de basuras, pérdidas por daños en las embarcaciones, altos costos en control de especies invasoras), que han sido identificados en diferentes estudios (Alimba \& Faggio, 2019; Andrady, 2011; Antão et al. 2018; Bennecke et al. 2016; ; Botterell et al. 2019; Bulow \& Ferdinand, 2013; Cruz \& Pérez, 2017; Giacomo et al. 2015; Guzzetti et al. 2018; Koelmans, 2015; Kovač et al. 2017; Kühn et al. 2015; Massos \& Tuner, 2017; Mohamed \& Obbard, 2014; Mouat et 
al. 2010; Newman et al. 2015; Prata et al. 2019b; Rech et al. 2016; Ríos et al. 2007; Sun et al. 2017; Wang et al. 2016; Wright et al. 2013).

Teniendo en cuenta que los manglares de la Ciénaga Grande de Santa Marta (CGSM) están contaminados por basura marina (Garcés-Ordóñez et al. 2019), el objetivo de este estudio fue identificar y evaluar los impactos negativos de la basura marina sobre el manglar en la CGSM. Estos resultados son útiles para generar conciencia sobre las consecuencias de la contaminación por basura marina y para dar sustento a la gestión ambiental necesaria para prevenir y reducir, significativamente, este tipo de contaminación en la CGSM y así contribuir a la recuperación del manglar y sus servicios ambientales.

\section{MATERIALES Y MÉTODOS}

Área de estudio. La CGSM es el sistema lagunar costero más productivo del Caribe colombiano (Fig. 1), declarado humedal RAMSAR, reserva biosfera y parque nacional (INVEMAR, 2018). Tiene un área aproximada de $4280 \mathrm{~km}^{2}$ y está formado por la planicie de inundación del río Magdalena, el complejo de ciénagas de Pajarales, el cuerpo de agua de la Ciénaga Grande y la Isla de Salamanca (INVEMAR, 2018). El clima es tropical árido, con una época seca y unalluviosa(IDEAM, 2014). Los mangles del bosque son: Rhizophora mangle, Laguncularia racemosa y Avicennia germinans (INVEMAR, 2018). En la CGSM se encuentran las cuales de 11 municipios, cuyas poblaciones desarrollan actividades de pesca artesanal, acuicultura, agroindustria y ganadería (INVEMAR \& MinAmbiente, 2013; Vilardy \& González, 2011).

Identificación de impactos. Se identificaron los impactos observados y potenciales de la contaminación por basura marina en los manglares de la CGSM, para lo que se tuvieron en cuenta las interacciones de la basura marina con elementos ambientales: aire, agua superficial, suelo del manglar, flora (mangles), fauna asociada,

Fig. 1. Mapa de localización geográfica de la Ciénaga Grande de Santa Marta, en el Caribe Colombiano. Los puntos rojos corresponden a las áreas de manglar donde se identificaron los impactos observados, los números en los puntos rojos están asociados a las fotografías que muestran la contaminación por basuras marinas en la población y dentro de los manglares Fig. 1. Geographic location of the Ciénaga Grande de Santa Marta, in the Colombian Caribbean. The red dots correspond to the mangrove areas where impacts were identified; numbers next red dots refer to the photographs showing marine litter pollution in the populated area and within the mangroves 


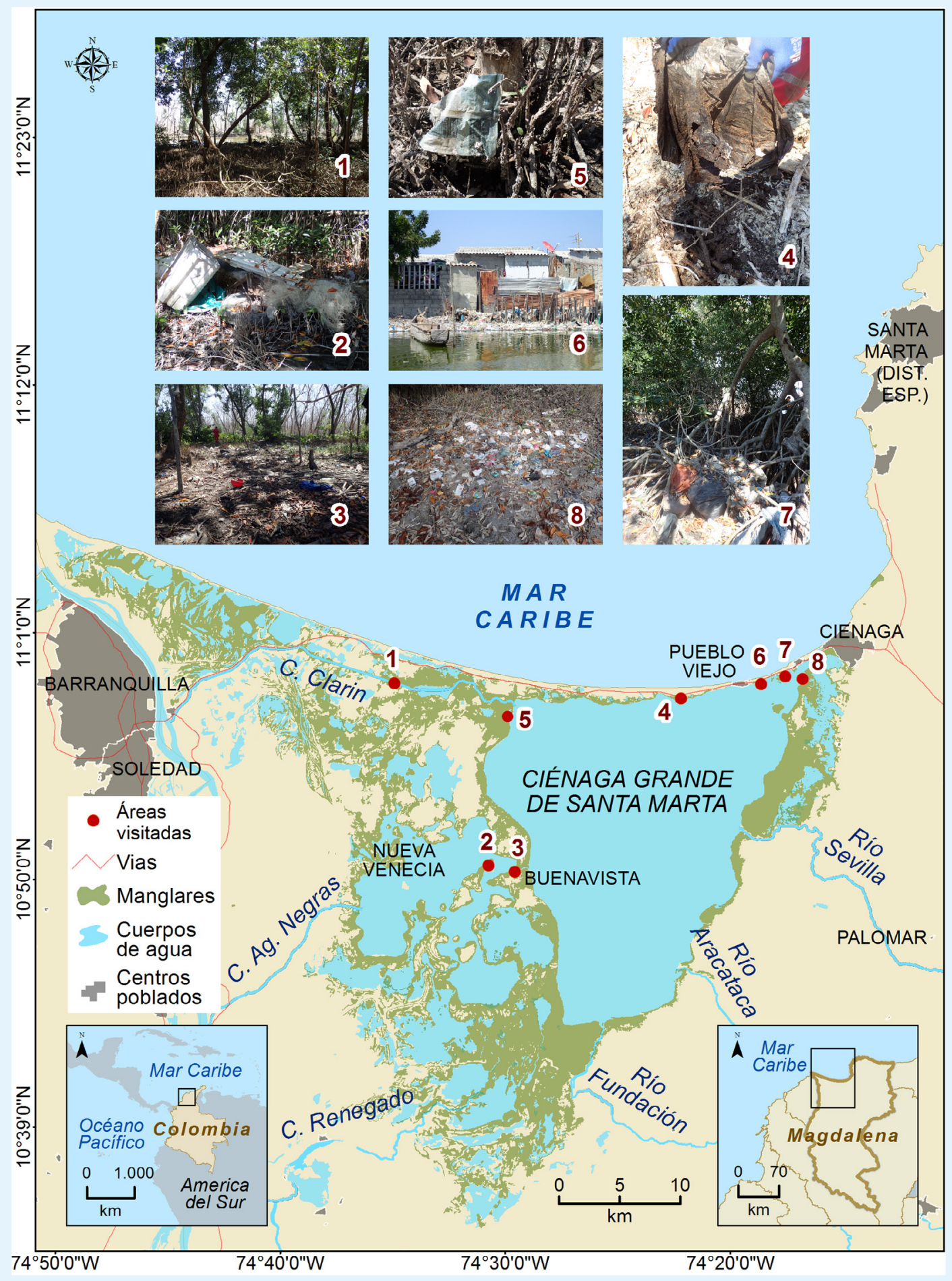


paisaje y comunidades humanas. Los impactos observados se evidenciaron directamente en ocho áreas de manglar ubicadas cerca (seis áreas) y lejos (dos áreas) de centros poblados de la CGSM, con concentraciones variadas de basura marina según Garcés-Ordóñez et al. (2019), durante las dos salidas de campo realizadas en este estudio en febrero de 2018. Los impactos potenciales resultaron de la revisión bibliográfica realizada, los cuales no se evidenciaron directamente en los manglares de la CGSM.

Para la identificación de los impactos potenciales se consultaron las bases de datos ScienceDirect, Springer Link y Google Académico, incluyendo las siguientes palabras clave en inglés y español: basura marina, contaminación por microplásticos, manglares e impacto de la basura marina. Se tomaron en cuenta las publicaciones realizadas entre los años 2000 y 2019 en idioma español e inglés, dándole mayor valor a los artículos y libros revisados por pares y reportes publicados por instituciones oficiales de alto prestigio. La búsqueda resultó en 83 documentos entre artículos, libros e informes técnicos, de los cuales se revisaron 55 documentos con información sobre los impactos físicos, químicos, biológicos y socioeconómicos de la basura marina. La identificación de los impactos potenciales es importante para alertar sobre los posibles riesgos a la salud del ecosistema y de la población humana de la CGSM, y servirá para la gestión, al respecto, de las autoridades ambientales nacionales y locales.

Valoración de impactos observados. Se utilizó como base la metodología propuesta por Fernández (2010), modificando la escala de calificación (Cuadro 1) e interpretación de siete criterios (intensidad -I, extensión -EX, momento -MO, reversibilidad $-\mathrm{RV}$, recuperabilidad -RP, sinergia -SI y acumulación -AC), para valorar los impactos de la basura marina observados directamente en los manglares de la CGSM, pues esta metodología está, desde su origen, diseñada para valorar impactos ambientales que podrían materializarse por causa de las actividades humanas. Para esta modificación se realizaron talleres y reuniones de trabajo con un grupo interdisciplinario de investigación y consultas a personal experto en el tema. A partir de ahí, se definió la interpretación de los criterios de la siguiente manera: 1) Intensidad: Grado de incidencia de la contaminación por basura marina sobre el manglar; 2) Extensión: Presencia del impacto en las ocho áreas visitadas; 3 ) Momento: Tiempo que transcurre entre la disposición de los residuos sólidos en el manglar y la aparición del impacto; 4) Reversibilidad: Posibilidades de retorno a la condición inicial sin implementar ninguna medida de manejo. 5) Recuperabilidad: Tiempo necesario para retornar a la condición inicial implementando medidas de manejo; 6) 
Sinergia: Posibilidad de que en com- del impacto, considerando una continua binación con otros eventos el impacto disposición de residuos sólidos en el pueda aumentarse significativamente; ecosistema.

7) Acumulación: Incremento progresivo

Cuadro 1. Escalas de valoración de los criterios propuestos por FernándezVítora (2010), adaptados para el presente estudio

Table 1. Assessment scales of the criteria proposed by Fernández-Vítora (2010), adapted for this study

\begin{tabular}{|c|c|c|}
\hline CRITERIO & CALIFICACIÓN & VALOR \\
\hline \multirow[t]{3}{*}{ Intensidad (IN) } & Afectación baja o mínima del aspecto ambiental & 1 \\
\hline & Afectación media del aspecto ambiental & 4 \\
\hline & Afectación alta del aspecto ambiental & 8 \\
\hline \multirow[t]{4}{*}{ Extensión (EX) } & Puntual ( 1 estación de muestreo) & 1 \\
\hline & Parcial (2 - 3 estaciones de muestreo) & 4 \\
\hline & Amplio o extenso ( 4 y 5 estaciones de muestreo) & 8 \\
\hline & Total (todas las estaciones de muestreo) & 12 \\
\hline \multirow[t]{4}{*}{ Momento (MO) } & Largo plazo ( $>3$ años) & 1 \\
\hline & Mediano plazo ( 1 - 3 años $)$ & 2 \\
\hline & Corto plazo $(<1$ año $)$ & 4 \\
\hline & Inmediato (tiempo mínimo o nulo) & 8 \\
\hline \multirow[t]{4}{*}{ Reversibilidad (RV) } & Corto plazo $(<1$ año $)$ & 1 \\
\hline & Mediano plazo (1 - 5 años) & 4 \\
\hline & Largo plazo ( $>5$ años $)$ & 8 \\
\hline & Irreversible & 12 \\
\hline \multirow{6}{*}{$\begin{array}{l}\text { Recuperabilidad } \\
\text { (RP) }\end{array}$} & De manera inmediata & 1 \\
\hline & A corto plazo $(<1$ año $)$ & 2 \\
\hline & A mediano plazo ( 1 - 3 años $)$ & 3 \\
\hline & A largo plazo ( $>3-14$ años $)$ & 4 \\
\hline & $\begin{array}{l}\text { Mitigable, sustituible y compensable (Sólo se recupera } \\
\text { parcialmente) }\end{array}$ & 8 \\
\hline & $\begin{array}{l}\text { Irrecuperable (No se recupera por medidas correctivas } \\
\text { ni acciones humanas) }\end{array}$ & 12 \\
\hline \multirow[t]{2}{*}{ Sinergia (SI) } & Sin sinergismo & 1 \\
\hline & Sinérgico & 8 \\
\hline \multirow[t]{2}{*}{ Acumulación (AC) } & Simple & 1 \\
\hline & Acumulativo & 8 \\
\hline
\end{tabular}


Debido a la disponibilidad de la información en la CGSM, solo se valoraron los impactos observados. Para establecer la importancia ambiental de cada impacto, se adaptó la fórmula de Fernández (2010) como sigue: Importancia $(\mathrm{I})=(3 \mathrm{IN}+2 \mathrm{EX}+\mathrm{MO}+\mathrm{RV}+\mathrm{R}-$ $\mathrm{P}+\mathrm{SI}+\mathrm{AC})$. Se partió de la premisa de que todos los impactos son negativos para la calidad ambiental y la salud del ecosistema de manglar. Establecida la fórmula y los valores para cada criterio, el valor mínimo posible por impacto fue de 10 y el máximo de 100. Los rangos de calificación cualitativa definidos se muestran en el Cuadro 2.

Cuadro 2. Rangos de calificación cualitativa de la importancia Ambiental del impacto

Table 2. Qualitative rating ranges of the environmental importance of the impact

\begin{tabular}{ll}
\hline Valor & Calificación del impacto \\
\hline 10 a 24 & Irrelevante \\
25 a 49 & Moderado \\
50 a 74 & Severo \\
75 a 100 & Critico \\
\hline
\end{tabular}

\section{RESULTADOS Y DISCUSIÓN}

Impactos observados. Se identificaron 10 impactos en los manglares de la CGSM. La calificación dada a cada criterio en los impactos observados y el resultado de su importancia ambiental se muestra en el Cuadro 3.
La contaminación por basura marina genera sobre el manglar de la CGSM impactos severos, moderados y críticos (Fig. 2). En el suelo, el impacto de mayor importancia fue la acumulación de microplásticos. La intensidad y la extensión de este impacto se evidencian en las concentraciones reportadas por Garcés-Ordóñez et al. (2019) en la CGSM, consideradas entre las más altas en manglares. Debido a las bajas tasas de degradación del plástico en condiciones naturales (Andrady, 2011) y a la constante disposición de residuos sólidos en el manglar, este impacto es acumulativo, y por el diminuto tamaño $(<5 \mathrm{~mm})$ de los microplásticos que al mezclarse con el suelo- impide su recuperación del ecosistema, es irreversible. Este se califica como crítico (Fig. 2).

La acumulación de plásticos en el suelo del manglar en la CGSM genera cambios físicos en las capas del suelo por su incorporación en los primeros horizontes, lo cual puede incidir en la aireación, movimiento del agua y en la regeneración natural (Sharma et al. 2018). Este impacto se observó en todas las áreas de manglar visitadas. Debido a la alta durabilidad, continua disposición del plástico y la baja resiliencia del ecosistema frente a estos contaminantes, se califica como un impacto severo (Fig. 2).

En el aire, se identificaron dos impactos moderados: aumento de 
Cuadro 3. Calificación dada a los impactos observados en los manglares de la Ciénaga Grande de Santa Marta. Intensidad (I), extensión (EX), momento (MO), reversibilidad (RV), recuperabilidad (RP), sinergia (SI) y acumulación (AC) e importancia (I)

Table 3. Rating given to the impacts observed in the mangroves of the Ciénaga Grande de Santa Marta. Intensity (I), extension (EX), momentum (MO), reversibility (RV), recoverability (RP), synergy (SI) and accumulation (AC) and importance (I)

\begin{tabular}{|c|c|c|c|c|c|c|c|c|c|c|}
\hline \multirow{2}{*}{$\begin{array}{l}\text { Elemento del } \\
\text { ecosistema }\end{array}$} & \multirow{2}{*}{$\begin{array}{l}\text { Aspecto } \\
\text { ambiental }\end{array}$} & \multirow[b]{2}{*}{ Impacto observado } & \multicolumn{7}{|c|}{ Criterio } & \multirow[b]{2}{*}{$\mathbf{I}$} \\
\hline & & & IN & EX & MO & RV & $\mathbf{R P}$ & $\mathrm{AC}$ & SI & \\
\hline \multirow{2}{*}{$\begin{array}{l}\text { AGUA } \\
\text { SUPERFICIAL }\end{array}$} & \multirow{2}{*}{$\begin{array}{l}\text { Calidad del } \\
\text { agua }\end{array}$} & $\begin{array}{l}\text { Acumulación de residuos } \\
\text { y microplásticos en la } \\
\text { columna del agua. }\end{array}$ & 4 & 8 & 4 & 12 & 4 & 8 & 8 & 64 \\
\hline & & $\begin{array}{l}\text { Aumento de sustancias } \\
\text { contaminantes en el agua. }\end{array}$ & 1 & 1 & 8 & 1 & 4 & 8 & 8 & 34 \\
\hline \multirow{2}{*}{ AIRE } & \multirow{2}{*}{$\begin{array}{l}\text { Calidad del } \\
\text { aire }\end{array}$} & $\begin{array}{l}\text { Generación de malos } \\
\text { olores. }\end{array}$ & 4 & 1 & 8 & 1 & 2 & 1 & 1 & 27 \\
\hline & & $\begin{array}{l}\text { Aumento en el material } \\
\text { particulado en el aire. }\end{array}$ & 4 & 1 & 2 & 1 & 3 & 1 & 8 & 29 \\
\hline \multirow{2}{*}{ SUELO } & $\begin{array}{l}\text { Calidad de } \\
\text { suelo }\end{array}$ & $\begin{array}{l}\text { Acumulación de partículas } \\
\text { de plástico en el suelo. }\end{array}$ & 8 & 12 & 4 & 12 & 8 & 8 & 1 & 81 \\
\hline & $\begin{array}{l}\text { Estructura } \\
\text { física }\end{array}$ & $\begin{array}{l}\text { Cambios en la estructura } \\
\text { física del suelo. }\end{array}$ & 4 & 12 & 2 & 8 & 8 & 8 & 1 & 63 \\
\hline \multirow[t]{2}{*}{$\begin{array}{l}\text { FLORA } \\
\text { (MANGLES) }\end{array}$} & $\begin{array}{l}\text { Regeneración } \\
\text { natural }\end{array}$ & $\begin{array}{l}\text { Limitación en el } \\
\text { establecimiento de } \\
\text { propágulos y crecimiento } \\
\text { de plántulas. }\end{array}$ & 4 & 4 & 4 & 8 & 3 & 8 & 8 & 51 \\
\hline & $\begin{array}{l}\text { Condiciones } \\
\text { fitosanitarias }\end{array}$ & $\begin{array}{l}\text { Daños físicos en las raíces } \\
\text { y ramas. }\end{array}$ & 1 & 12 & 4 & 4 & 8 & 1 & 1 & 45 \\
\hline $\begin{array}{l}\text { FAUNA } \\
\text { ASOCIADA }\end{array}$ & Hábitat & $\begin{array}{l}\text { Cambios en la } \\
\text { disponibilidad de hábitats. }\end{array}$ & 8 & 12 & 2 & 8 & 3 & 8 & 1 & 70 \\
\hline PAISAJE & Calidad visual & $\begin{array}{l}\text { Deterioro paisajístico por } \\
\text { acumulación de residuos. }\end{array}$ & 1 & 12 & 4 & 8 & 3 & 8 & 1 & 51 \\
\hline
\end{tabular}

partículas suspendidas y generación de malos olores (Fig. 2). El primero obedece al desprendimiento de microplásticos, partículas de polvo y fibras de tela generadas de la degradación de la basura marina, las cuales pueden quedar suspendidas en un tiempo determinado y precipitarse nuevamente, 


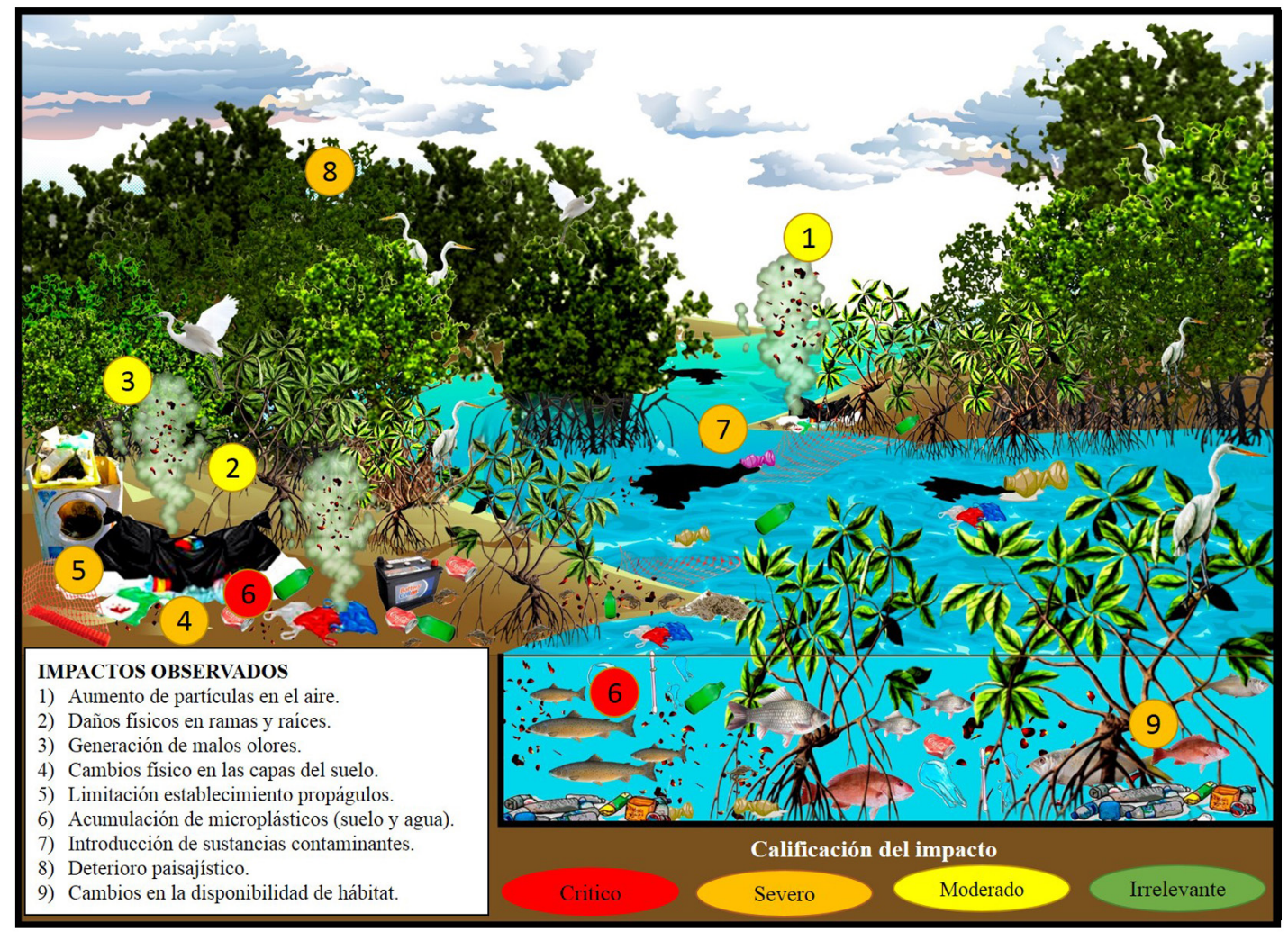

Fig. 2. Impactos ambientales observados en el manglar a causa de la presencia, acumulación y degradación de la basura marina en la CGSM. Impactos en color rojo son críticos, en naranja son severos, en amarillo son moderados y en verde son irrelevantes. Fuente: Elaboración propia

Fig. 2. Environmental impacts observed in mangroves due to the presence, accumulation, and degradation of marine debris in the CGSM. Impacts in red are consider critical, orange severe, yellow moderate, and green irrelevant. Source: Own elaboration

o, dependiendo de la temporada climática, pueden ser llevadas a otros sitios por acción de los vientos Alisios que se presentan especialmente entre diciembre y abril (Arévalo \& Franco, 2008). Si bien es un impacto reversible debido a la precipitación de las partículas sus- pendidas, es calificado como moderado, ya que, en combinación con otras actividades humanas como el transporte terrestre, proyectos de infraestructura y las mismas condiciones del ambiente, pueden generar una mayor contaminación (CORPAMAG, 2018). 
El segundo (la generación de malos olores en el ambiente) se produce por la disposición de material orgánico y residuos sanitarios como pañales desechables y toallas higiénicas, los cuales pueden llegar a ser intensos, dependiendo de la densidad de basura acumulada. Estos residuos se observaron en el manglar cercano a las poblaciones humanas en la CGSM, donde este se usa como botadero de basura.

En las aguas superficiales cercanas al manglar se apreciaron y evaluaron dos impactos. El primero es la acumulación de macrobasuras (con tamaño $>5 \mathrm{~mm}$ ) y microplásticos en la columna del agua (Fig. 2). Este se consideró irreversible sin implementación de medidas de manejo; recuperable parcialmente considerando el tamaño de las partículas de plástico; sinérgico si se determina que no solo los residuos sólidos afectan la calidad del agua y, además, acumulativo; ya que, al ser la CGSM un sistema cerrado, en condiciones climáticas de altas precipitaciones se presentan grandes inundaciones y apertura del sector donde se conectan la CGSM con el mar Caribe (INVEMAR, 2018). Además, cabe resaltar las implicaciones que la basura marina tiene sobre la fauna acuática (ver impactos potenciales); por estas condiciones, se califica como severo.

El segundo impacto corresponde a la introducción de sustancias contaminantes en el agua (Fig. 2), observa- do solo en una de las zonas visitadas, próxima a la población de Isla del Rosario, donde se encontraron envases con residuos oleosos y se observó iridiscencia en el agua adyacente de los mangles. Este impacto se considera acumulativo y sinérgico con otras actividades como el vertimiento de aguas residuales y la navegación con motor a bordo (Vivas-Aguas et al. 2013), por lo cual se califica como moderado.

En el manglar, la presencia de basura marina en el suelo y la basura enredada en las plantas limita el establecimiento de los propágulos y crecimiento normal de las plántulas (Fig. 2), lo que puede afectar la regeneración del manglar de la CGSM, por la sinergia con la alta salinidad intersticial (entre 60 y 90 UPS) de los últimos años, por el efecto del evento El Niño que causa sequías y estrés a las plantas (INVEMAR, 2018). Adicionalmente, este impacto es acumulativo, por la continuidad de la disposición de basura marina en el manglar; sin embargo, puede ser recuperable en el mediano plazo (1-5 años) con medidas de limpieza eficientes y de prevención. Este se califica como severo.

Los daños físicos en las raíces y ramas de los mangles (Fig. 2) se observaron en todas las áreas de manglar visitadas. En el área cercana al centro poblado Buenavista, se encontraron electrodomésticos, muebles, sanitarios de cerámica, láminas metálicas oxidadas y redes de pesca que generan 
presión y causan daños en los neumatóforos de A. germinans y L. racemosa. En otras áreas visitadas se encontró basura enredada en las raíces de $R$. mangle y $A$. germinans, que causaron deformación. Este impacto se califica como moderado.

En la fauna se identificó el cambio en la calidad del hábitat (Fig. 2), el cual se califica como impacto severo por su extensión, acumulación y reversibilidad; afecta sobre todo a los organismos invertebrados que habitan en las raíces de los mangles y en el suelo: cangrejos, cirrípedos, caracoles, entre otros. Los invertebrados son la base fundamental en la red trófica del manglar (Margalef, 1980), por lo cual es necesario realizar estudios detallados que permitan establecer los efectos de la basura marina sobre estas poblaciones.

Finalmente, el deterioro paisajístico por acumulación de basura (Fig. 2) fue evidente a lo largo del manglar de la CGSM. Incluso, la primera impresión, al acercarse al complejo lagunar de la CGSM, es la apariencia de un ecosistema con una salud pobre y sometida frecuentemente a los diferentes factores de degradación (Vivas-Aguas et al. 2013). Esto tendrá repercusión no solo sobre la calidad ambiental del manglar, sino también sobre la posibilidad de generar actividades económicas alternativas a la pesca, como el ecoturismo. Por estas razones se califica como severo.
Impactos potenciales. Se identificaron en total 11 impactos potenciales. Entre los cuales está el detrimento de la regeneración natural del manglar de la CGSM (Fig. 3). Este se puede presentar como consecuencia de la acumulación de basura marina en el suelo, lo cual forma una barrera que puede dificultar el desarrollo de propágulos y de plántulas, por limitar el crecimiento de las raíces. Adicionalmente, la basura marina arrojada sobre estos causarles daños físicos, aplastamiento y muerte. Este impacto puede tener sinergia con otros factores de degradación ambiental reportados para la CGSM, como la elevada salinidad y herbivorismo (INVEMAR, 2018).

La obstrucción del intercambio gaseoso entre los mangles y la atmósfera (Fig. 3) también se identificó como impacto potencial, ya que, en las áreas de manglar visitadas, se encontró basura marina depositada sobre los neumatóforos de $L$. racemosa y $A$. germinans, y sobre las raíces fúlcrea de $R$. mangle. Esta condición puede tener sinergia con condiciones de alta inundación, magnificar el impacto $\mathrm{y}$ provocar alteraciones fisiológicas en los mangles.

El ingreso y acumulación de sustancias contaminantes en el suelo del manglar de la CGSM (Fig. 3) se consideró un impacto potencial por la presencia de residuos peligrosos 


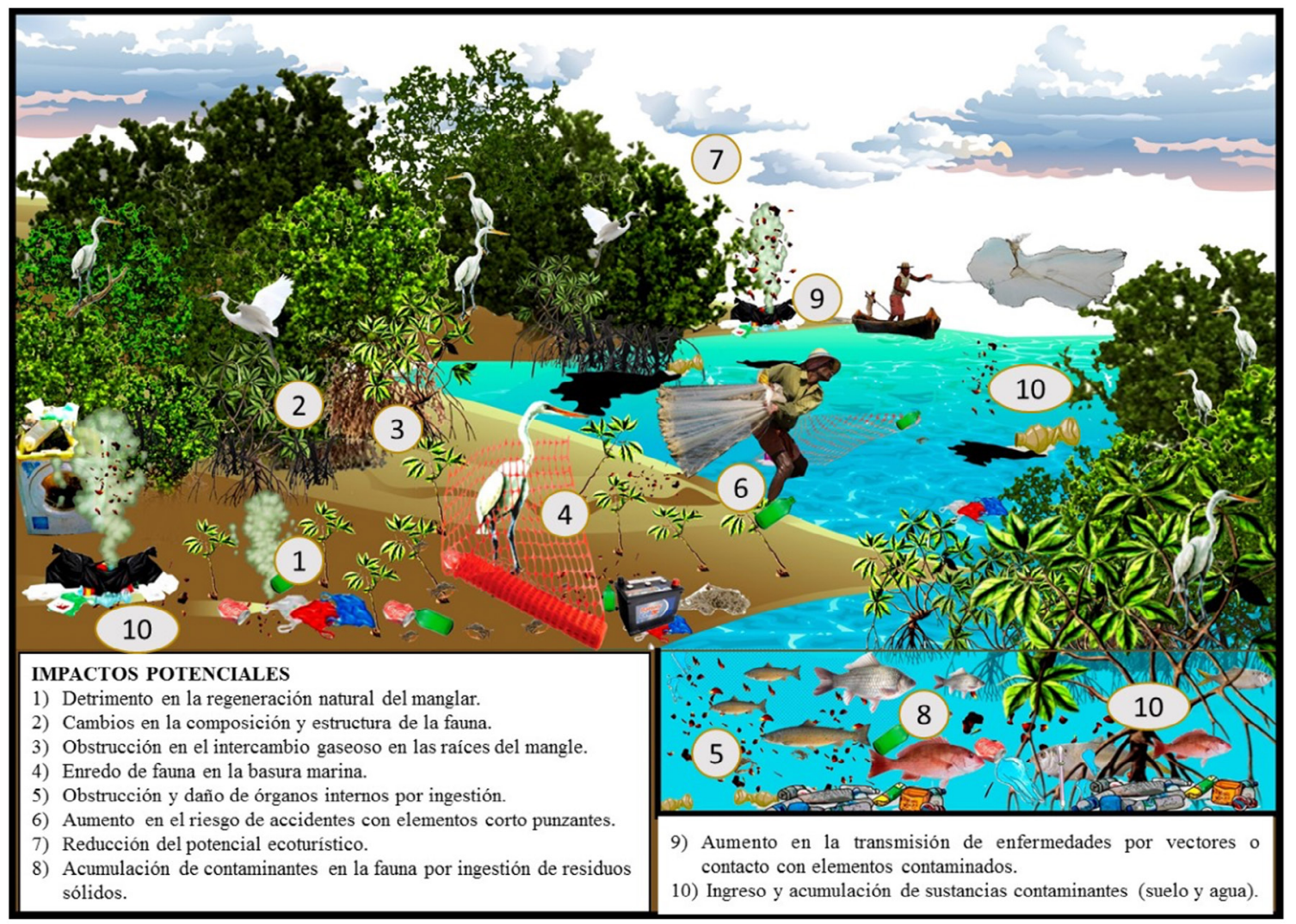

Fig. 3. Impactos ambientales potenciales en el manglar de la CGSM a causa de la presencia, acumulación y degradación de la basura marina. Fuente: Elaboración propia

Fig. 3. Potential environmental impacts in CGSM mangroves due to the presence, accumulation, and degradation of marine debris. Source: Own elaboration

como envases de aceite para motor, plaguicidas y pinturas, baterías AA, medicamentos, bombillas y luminarias (Garcés-Ordóñez et al. 2019). Estos residuos pueden liberar, al medio, sustancias tóxicas como hidrocarburos aromáticos policíclicos, plaguicidas y metales pesados, los cuales contaminan el suelo y el agua, y deterioran su calidad (Andrady, 2011; Koelmans, 2015; Wang et al. 2016). Adicionalmente, estos contaminantes pueden ser adsorbidos por los mangles a través de sus raíces, se pueden bioacumular en los tejidos vegetales e ingresar a la red trófica a través de 
herbívoros y detritívoros (Arumugam et al. 2018; Li et al. 2019). Esto representaría, según la concentración, un riesgo de efectos adversos en la salud de especies de crustáceos, moluscos, reptiles, aves y peces asociados al manglar e, incluso, a la población humana que también puede afectarse por la ingestión de alimentos contaminados (Giacomo et al. 2015; Guzzetti et al. 2018; Ríos et al. 2007).

La fauna del manglar de la CGSM también puede impactarse negativamente, al enredarse con la basura marina durante su desplazamiento y búsqueda de alimentos (Fig. 3). Las aves, los crustáceos, reptiles y peces podrían ser los grupos de animales más vulnerables. Entre los daños físicos que podrían producirse por el enredo están el estrangulamiento, deformaciones, amputación de partes del cuerpo, ahogamiento, reducción de la supervivencia y la muerte (Kühn et al. 2015; Wang et al. 2016). Las redes de pesca elaboradas con nylon y las bolsas plásticas observadas en los manglares de la CGSM tienen mayor posibilidad de causar daños por enredo a la fauna, debido a sus múltiples formas y abundancia en el medio.

Otro impacto potencial para la fauna del manglar de la CGSM es la obstrucción y daño de órganos internos (Fig. 3), por respiración de microplásticos, por la ingestión de forma accidental o por confusión de la basura marina con alimento (Ory et al.
2017; Sun et al. 2017). Los peces, los moluscos y los crustáceos de la CGSM pueden ser los de mayor incidencia en la ingestión y respiración de estos contaminantes; sin embargo, esta hipótesis requiere ser comprobada con la investigación. Como consecuencia de la obstrucción del tracto digestivo o respiratorios, se pueden presentar daños en órganos internos, reducir el crecimiento y desarrollo, alterar las funciones fisiológicas y del sistema inmunológico, reducir la supervivencia y producir, por último, la muerte (Kühn et al. 2015; Prata et al. 2019b). Con la ingestión de basura marina, los animales pueden adsorber hidrocarburos aromáticos policíclicos; plaguicidas organoclorados; aditivos del plástico como el nonilfenol, bisfenol A, ftalatos, polibromodifenil éteres y metales pesados, que también son introducidos en la red trófica; causar efectos adversos (alteraciones en respuestas inmunológicas, disrupción endocrina con repercusiones en la reproducción y desarrollo, cáncer o mutagénesis) en los organismos acuáticos $\mathrm{y}$, posiblemente, afectar la salud de la comunidad humana local por ingerir alimentos contaminados (Andrady, 2011; Giacomo et al. 2015; Koelmans, 2015; Ohore \& Zhang, 2019; Prata et al. 2019b; Ríos et al. 2007; Wang et al. 2016).

Se pueden presentar cambios en la composición y estructura de la comunidad faunística del manglar de la CGSM (Fig. 3) como consecuencia del 
deterioro del hábitat, ya que las especies más sensibles a la contaminación podrían desplazarse hacia otros manglares, quedando posiblemente las especies más resistentes o indiferentes a la contaminación por la basura marina. En el caso de pequeños invertebrados como cangrejos, se ha reportado, para otros manglares, que la acumulación de basura inhibe las actividades normales que estos invertebrados realizan y limita el suelo físico requerido para la realización de sus madrigueras $(\mathrm{Bu}-$ low \& Ferdinand, 2013); esto podría presentarse en los manglares cercanos a los centros poblados de la CGSM donde se está acumulando una gran cantidad de basura. Lo anterior propiciaría cambios en la estructura de estas comunidades y pérdida de funciones ecológicas de las especies en el ecosistema (Kristensen, 2008).

Como impacto potencial negativo para la comunidad de la CGSM, se identificó el aumento del riesgo de accidentes con elementos corto punzantes (Fig. 3). Los residuos como vidrios, jeringas, frascos metálicos y fragmentos de plástico duro pueden lastimar y causar heridas a pescadores o personas que extraen madera para uso doméstico. Estas heridas y la presencia de microorganismos de origen fecal en el agua, por los constantes vertimientos de aguas residuales sanitarias (INVEMAR, 2018), aumentan el riesgo de infecciones por microorganismos patógenos (Ballesteros et al.
2005; Greenpeace, 2005; Pukkala \& Ponka, 2001). Por último, se identificó la reducción del potencial ecoturístico por la acumulación de basura marina, el deterioro de la calidad ambiental del manglar y detrimento de la belleza paisajística de la CGSM. Otras actividades como la pesca y la navegación pueden verse afectadas por la basura marina y otros contaminantes que causan disminución de abundancias del recurso pesquero y pérdidas económicas de la población de la CGSM (Greenpeace, 2005; Mouat et al. 2010).

Recomendaciones para la gestión ambiental en la CGSM. Con base en los impactos identificados en este estudio, se recomiendan las siguientes acciones para mejorar la gestión ambiental:

1) Planificar participativamente el desarrollo social y económico, y promover la conservación ambiental en la ecorregión CGSM, considerando los principios del desarrollo sostenible (Moller, 2010).

2) Diseñar e implementar estrategias de recolección participativa de residuos sólidos en las comunidades de la CGSM, en las cuales se promuevan actividades de reciclaje y aprovechamiento lideradas por la comunidad.

3) Diseñar e implementar sistemas de tratamiento de aguas residuales para las poblaciones palafíticas de la CGSM. 
4) Fortalecer los procesos de educación ambiental enfocados hacia la prevención de la contaminación por basura marina, dirigida a cada grupo social (pescadores, jóvenes, amas de casa) en las comunidades.

5) Realizar jornadas participativas de recolección de basura marina en los manglares de la CGSM.

6) Ampliar el conocimiento sobre los impactos y efectos de la basura marina en los ecosistemas y comunidades humanas de la CGSM.

Estas acciones implican la participación de los diferentes entes gubernamentales, la academia, los institutos de investigaciones y las comunidades de la CGSM para la planificación y gestión ambiental, que lleve al fortalecimiento del saneamiento básico, la generación de medidas para el control y seguimiento, la investigación científica y el desarrollo de acciones correctivas y compensatorias, para conocer los efectos, tomar conciencia del problema y reducir significativamente el ingreso de basura marina a la CGSM.

\section{CONCLUSIONES}

La presencia, la acumulación y la degradación de basura marina generan impactos moderados, severos y críticos en el manglar de la CGSM, de los cuales se resaltan, por su importancia, la acumulación de microplásticos en el ambiente, la limitación del establecimiento de propágulos y crecimiento de plántulas, y los cambios físicos en las capas de suelo.

Estos impactos desencadenan impactos potenciales sobre la calidad del hábitat, la fauna, la flora y las comunidades humanas de la CGSM. Dado el alto estado de deterioro del manglar en este complejo lagunar y la alta dependencia de la población humana local a los servicios ecosistémicos brindado por la CGSM, estos requieren ser investigados con mayor detalle y prontitud.

Las acciones para el manejo del problema de la basura marina en la CGSM deben estar orientadas hacia el ordenamiento territorial, las buenas prácticas de cada actividad económica, la educación y sensibilización ambiental, la limpieza de basura y la investigación; además, deben involucrar, activamente, a la comunidad en el diseño, formulación y aplicación de cada medida, para fortalecer, así, la gobernanza y promover la conservación del ecosistema.

\section{AGRADECIMIENTOS}

Este trabajo fue financiado por el Ministerio de Ambiente y Desarro1lo Sostenible, Colombia. Los autores agradecen al Instituto de Investigaciones Marinas y Costeras “José Benito 
Vives de Andréis" -INVEMAR por el apoyo institucional, al investigador $\mathrm{Fe}$ lipe Valencia Ospina por el apoyo cartográfico y al diseñador gráfico Mauro Robles por el diseño de las figuras de impactos observados y potenciales. Contribución científica del INVEMAR No. 1239. Y a los evaluadores anónimos que ayudaron a contribuir a este trabajo.

\section{BIBLIOGRAFÍA}

Alimba, C. \& Faggio, C. (2019). Microplastics in the marine environment: Current trends in environmental pollution and mechanisms of toxicological profile. Environ. Toxicol. Pharmacol., 68, 61-74. https://doi.org/10.1016/j. etap.2019.03.001

Andrady, A. (2011). Microplastic in the marine environment. Mar. Pollut. Bull., 62(8), 1596-1605. https://doi.org/10.1016/j. marpolbul.2011.05.030

Antão, L., Vethaak, A., Lavorante, B., Lundebye, A. \& Guilhermino, L. (2018). Marine microplastic debris: An emerging issue for food security, food safety and human health. Mar. Pollut. Bull., 133, 336-348. https://doi. org/10.1016/j.marpolbul.2018.05.047

Arévalo,D.\&Franco,A.(2008). Características oceanográficas de la surgencia frente a la ensenada de Gaira, departamento de Magdalena, época seca menor de 2006. Bol. Invest. Mar. Cost., 37(2), 131162. https://doi.org/10.25268/bimc. invemar.2008.37.2.195

Arumugam, A., Rajendran, R., Ganesan, A. \& Sethu, R. (2018). Bioaccumulation and translocation of heavy metals in mangrove rhizosphere sediments to tissues of Avicennia marina - A field study from tropical mangrove forest. ENMM, 10, 272-279. https://doi. org/10.1016/j.enmm.2018.07.005

Ballesteros, V., Cuadros, Y., Botero, S. \& López, Y. (2005). Factores de riesgo biológicos en recicladores informales de la ciudad de Medellín, 2005. Rev. Fac. Nac. Salud Pública, 26(2), 169177.

Bennecke, D., Duarte, B., Paiva, F., Cacador, I. \& Canning-Clode, J. (2016). Microplastic as vector of heavy metal contamination from the marine environment. Estuar. Coast. Shelf Sci., 178, 189-195. https://doi.org/10.1016/j. ecss.2015.12.003

Botterell, Z., Beaumont, N., Dorrington, T., Steinke, M., Thompson, R. \& Lindeque, P. (2019). Bioavailability and effects of microplastics on marine zooplankton: A review. Environ. Pollut., 245, 98-110. https://doi. org/10.1016/j.envpol.2018.10.065

Bulow, E. \& Ferdinand, T. (2013). El efecto de la basura en la dinámica de los ecosistemas de manglar: Un análisis comparativo. Ciudad de Panamá, Rep. Panamá: Smithsonian Institute y McGill University.

Cordero, C. \& Costa, T. (2010). Evaluation of solid residues removed from a mangrove swamp in the São Vicente Estuary, SP, Brazil. Mar. Pollut. Bull., 60(10), 1762-1767. https://doi. org/10.1016/j.marpolbul.2010.06.010

CORPAMAG. (2018). Informes de calidad de aire en el departamento del Magdalena. Recuperado de https://www.corpamag. gov.co/infousers/InfoSVCA.php

Cruz, Y. \& Pérez, O. (2017). Evaluación de impactos a la salud del manglar en el municipio de Guamá, Santiago de Cuba, Cuba. Madera Bosques, 23(1), 23-37. https://doi.org/10.21829/ myb.2017.2311517 
Debrot, O., Bron, P. \& León, R. (2013). Marine debris in mangroves and on the seabed: Largely-neglected litter problems. Mar. Pollut. Bull., 72, 1. https://doi. org/10.1016/j.marpolbul.2013.03.023

Fernández, V.C. (2010). Guía metodológica para la evaluación del impacto ambiental. Madrid, España: Ediciones Mundi-Prensa.

Foulon, V., Le Roux F., Lambert C., Huvet A., Soudant P. \& Paul-Pont I. (2016). Colonization of polystyrene microparticles by Vibrio crassostreae: light and electron microscopic investigation. Environ. Sci. Technol., 50(20), 10988-10996. https://doi. org/10.1021/acs.est.6b02720

Garcés-Ordóñez, O., Castillo-Olaya, V., Granados-Briceño, A., Blandón, L. \& Espinosa, L. (2019). Marine litter and microplastic pollution on mangrove soils of the Ciénaga Grande de Santa Marta, Colombian Caribbean. Mar. Pollut. Bull., 145, 455-462. https://doi. org/10.1016/j.marpolbul.2019.06.058

Garcés-Ordóñez, O., Castillo, V., Rueda, R., Ríos M., Bayona, M., Molina, F. \& Escobar, M. (2017). Diagnóstico de residuos microplásticos en las zonas marinas de Colombia. En INVEMAR \& MINAMBIENTE, Formulación de lineamientos, medidas de conservación, manejo y uso de ecosistemas marinos y costeros, con la intención de apoyar acciones de fortalecimiento en la gestión ambiental de las zonas costeras de Colombia (pp. 108-166). Santa Marta, Colombia: INVEMAR.

Giacomo, C., Gorbi, S., Milan, M., Benedetti, M., Fattorini, D., d'Errico, G., Pauletto, M., Bargelloni, L. \& Regoli, F. (2015). Pollutants bioavailability and toxicological risk from microplastics to marine mussels. Environ. Pollut., 198,
211-222. https://doi.org/10.1016/j. envpol.2014.12.021

Greenpeace. (2005). Basuras en el mar. Madrid, España: Greenpeace.

Guzzetti, E., Sureda, A., Tejada, S. \& Faggio, C. (2018). Microplastic in marine organism: Environmental and toxicological effects. Environ. Toxicol. Pharmacol., 64, 164-171. https://doi. org/10.1016/j.etap.2018.10.009

Horton, A., Walton, A., Spurgeon, D. J., Lahive, E. \& Svendsen, C. (2017). Microplastics in freshwater and terrestrial environments: Evaluating the current understanding to identify the knowledge gaps and future research priorities. Sci. Total Environ., 586, 127-141. https://doi.org/10.1016/j. scitotenv.2017.01.190

IDEAM. (2014). Atlas climatológico de Colombia 1981-2010. Recuperado de http://atlas.ideam.gov.co/ visorAtlasClimatologico.html

INVEMAR. (2018). Monitoreo de las condiciones ambientales y los cambios estructurales y funcionales de las comunidades vegetales y de los recursos pesqueros durante la rehabilitación de la Ciénaga Grande de Santa Marta (Informe Técnico Final 2017). Santa Marta: Colombia: INVEMAR.

INVEMAR \& MinAmbiente. (2013). Elementos técnicos y generación de capacidad para el ordenamiento $y$ manejo de los espacios y recursos marinos, costeros e insulares de Colombia. Santa Marta, Colombia.: INVEMAR.

Iñiguez, M. E., Conesa, J. A. \& Fullana, A. (2016). Marine debris ocurrence and treatment: A review. Renew Sust Energ Rev., 64, 394-402. https://doi. org/10.1016/j.rser.2016.06.031

Ivar do Sul, J., Costa, M., Silva-Cavalcanti, J. \& Araújo, M. (2014). Plastic 
debris retention and exportation by a mangrove forest parch. Mar. Pollut. Bull., 78(1-2), 252-257. https://doi. org/10.1016/j.marpolbul.2013.11.011

Jambeck, J., Geyer, R., Wilcox, C., Siegler, T., Perryman, M., Andrady, A., Narayan, R. \& Lavender, K. (2015). Plastic waste inputs from land into the ocean. Science, 347(6223), 768-771. https:// doi.org/10.1126/science.1260352

Kirstein, I.V., Kirmizi, S., Wichels, A., GarinFernandez, A., Erler, R., Löder, M. \& Gerdts, G. (2016). Dangerous hitchhikers? Evidence for potentially pathogenic Vibrio spp. on microplastic particles. Mar. Environ. Res., 120, 1-8. $\quad$ https://doi.org/10.1016/j. marenvres.2016.07.004

Koelmans, A. (2015). Modeling the Role of Microplastics in Bioaccumulation of Organic Chemicals to Marine Aquatic Organisms. A Critical Review. En M. Bergmann, L. Gutow \& M. Klages (Eds.), Marine Anthropogenic Litter (pp. 309-324). Berlin, Germany: Springer.

Kovač, M., Nika, M., Koren, Š., Kržan, A. \& Peterlin, M. (2017). Microplastics as a vector for the transport of the bacterial fish pathogen species Aeromonas salmonicida. Mar. Pollut. Bull., 125(12), 301-309. https://doi.org/10.1016/j. marpolbul.2017.08.024

Kristensen, E. (2008). Mangrove crabs as ecosystem engineers; with emphasis on sediment processes. J. Sea Res., 59(12), 30-43. https://doi.org/10.1016/j. seares.2007.05.004

Kühn, S., Bravo, E. L. \& Van Franeker, J. A. (2015). Deleterious effects of litter on marine life. En M. Bergmann, L. Gutow \& M. Klages (Eds.), Marine Anthropogenic Litter (pp. 75-116). Berlin, Germany: Springer. https://doi. org/10.1007/978-3-319-16510-3 4
Lebreton, L., van der Zwet, J., Damsteeg, J., Slat, B., Andrady, A. \& Reisser, J. (2017). River plastic emissions to the world's oceans. Nat. Commun., 8, 15611. https://doi.org/10.1038/ ncomms 15611

Li, H., Hu, Y., Sun, Y., De Silva, A.O., Muir, D., Wang, W., Xie, J., Xu, X., Pei, N., Xiong, Y., Luo, X. \& Mai, B. (2019). Bioaccumulation and translocation of tetrabromobisphenol A and hexabromocyclododecanes in mangrove plants from a national nature reserve of Shenzhen City, South China. Environ. Internat., 129, 239-246. https://doi.org/10.1016/j. envint.2019.05.034

Li, J., Zhang, H., Zhang, K., Yang, R., Li, R. \& Li, Y. (2018). Characterization, source, and retention of microplastic in sandy beaches and mangrove wetlands of the Qinzhou Bay, China. Mar. Pollut. Bull., 136, 401-406. https://doi.org/10.1016/j. marpolbul.2018.09.025

Margalef, R. (1980). Ecología. Barcelona, España: Editorial Omega.

Martin, C., Almahasheer, H. \& Duarte, C. (2019). Mangroves forests as traps for marine litter. Environ. Pollut., 247, 499-508. https://doi.org/10.1016/j. envpol.2019.01.067

Massos, A. \& Turner, A. (2017). Cadmium, lead, and bromine in beached microplastics. Environ. Pollut., 227, 139-145. https://doi.org/10.1016/j. envpol.2017.04.034

Mohamed, N. \& Obbard, J. (2014). Microplástics in Singapore's coastal mangrove ecosystem. Mar. Pollut. Bull., 79(1-2), 278-283. https://doi. org/10.1016/j.marpolbul.2013.11.025

Moller, R. (2010). Principios de desarrollo sostenible para América Latina. Ing. Recur. Nat. Amb., 9, 101- 
$110 . \quad$ https://www.redalyc.org/ pdf/2311/231116434012.pdf

Mouat, J., López, R. \& Bateson, H. (2010). Economic impacts of marine litter. Lerwick, UK: KIMO.

Naji, A., Esmaili, Z., Mason, S. \& Vethaak, A. D. (2017). The occurrence of microplastic contamination in littoral sediments of the Persian Gulf Iran. ESPR, 24(25), 20459-20468. https:// doi.org/10.1007/s11356-017-9587-z

Newman, S., Watkins, E., Farmer, A., Brink, P. \& Schweitzer JP. (2015). The Economics of Marine Litter. En M. Bergmann, L. Gutow \& M. Klages (Eds), Marine Anthropogenic Litter (pp 367-394). Cham, Alemania: Springer. https://doi.org/10.1007/978-3-31916510-3_14

Novotna, K., Cermakova, L., Pivokonska, L., Cajthaml, T. \& Pivokonsky, M. (2019). Microplastics in drinking water treatment - Current knowledge and research needs. Sci. Total Environ., 667, 30-740. https://doi.org/10.1016/j. scitotenv.2019.02.431

Ohore, O. E. \& Zhang, S. (2019). Endocrine disrupting effects of bisphenol A exposure and recent advances on its removal by water treatment systems. A review. Scient. African, 5, e00135. https://doi.org/10.1016/j.sciaf.2019. e00135

Ory, N., Sobral P., Ferreira J. \& Thiel M. (2017). Amberstripe scad Decapterus muroadsi (Carangidae) fish ingest blue microplastics resembling their copepod prey along the coast of Rapa Nui (Easter Island) in the South Pacific subtropical gyre. Sci. Total Environ., 586, 430-437. https://doi.org/10.1016/j. scitotenv.2017.01.175

Prata, J. C., da Costa, J. P., Lopes, I., Duarte, A. C. \& Rocha-Santos, T. (2019a). Effects of microplastics on microalgae populations: A critical review. Sci. Total Environ., 665, 400-405. https://doi. org/10.1016/j.scitotenv.2019.02.132

Prata, J. C., da Costa, J. P., Lopes, I., Duarte A. C., \& Rocha-Santos, T. (2019b). Environmental exposure to microplastics: an overview on possible human health effects. Sci. Total Environ., 134455 (en prensa). $\quad$ https://doi.org/10.1016/j. scitotenv.2019.134455

Pukkala, E. \& Ponka, A. (2001). Increased Incidence of Cancer and Asthma in Houses Built on a Former Dump Area. Environ. Health Perspect., 109(11), 1121-1125. https://doi.org/10.1289/ ehp.011091121

Rainieri, S. \& Barranco, A. (2019). Microplastics, a food safety issue? Trends Food Sci. Technol., 84, 55-57. https://doi.org/10.1016/j. tifs. 2018.12.009

Rech, S., Borrell, Y. \& García-Vázquez, E. (2016). Marine litter as vector of non-native especies: what we need to know. Mar. Pollut. Bull., 113(12), 40-43. https://doi.org/10.1016/j. marpolbul.2016.08.032

Riascos, J., Valencia, N., Peña, E. \& Cantera, J. (2019). Inhabiting the technosphere: The encroachment of anthropogenic marine litter in Neotropical mangrove forests and its use as habitat by macrobenthic biota. Mar. Pollut. Bull., 142, 559-568. https://doi.org/10.1016/j. marpolbul.2019.04.010

Ríos, L.M., Moore, C. \& Jones, P.R. (2007). Persistent organic pollutants carried by synthetic polymersin the ocean environment. Mar. Pollut. Bull., 54(8), 1230-1237. https://doi.org/10.1016/j. marpolbul.2007.03.022

Sharma, A., Kumar, A. \& Ganguly, R. (2018). Impact of open dumping of municipal solid waste on soil properties in 
mountainous region. $J$ Rock Mech. Geotech. Eng., 10(4), 725-739. https:// doi.org/10.1016/j.jrmge.2017.12.009

Smith, S. (2012). Marine debris: A proximate threat to marine sustainability in Bootless Bay, Papua New Guinea. Marine. Mar. Pollut. Bull., 64, 18801883. https://doi.org/10.1016/j. marpolbul.2012.06.013

Sun, X., Li, Q., Zhu, M., Liang, J., Zheng, S. \& Zhao, Y. (2017). Ingestion of microplastic by natural groups in the northern South China Sea. Mar. Pollut. Bull., 115(1-2), 217-224. https://doi. org/10.1016/j.marpolbul.2016.12.004

Troost, T. A., Desclaux, T., Leslie, H. A., van Der Meulen, M. D. \& Vethaak, A. D. (2018). Do microplastics affect marine ecosystem productivity? Mar. Pollut. Bull., 135, 17-29. https://doi. org/10.1016/j.marpolbul.2018.05.067

UNEP \& GRID-Arendal. (2016). Marine Litter Vital Graphics. Nairobi, Kenia: UNEP y GRID.

Vilardy, S. \& González, J. (Eds.). (2011). Repensando la Ciénaga: Nuevas miradas y estrategias para la sostenibilidad en la Ciénaga Grande de Santa Marta. Santa Marta, Colombia: Universidad del Magdalena y Universidad Autónoma de Madrid.
Vivas-Aguas, L. J., Espinosa, L. F. \& Parra, L. (2013). Identificación de fuentes terrestres de contaminación y cálculo de las cargas de contaminantes en el área de influencia de la Ciénaga Grande de Santa Marta, Caribe colombiano. Bol. Investig. Mar. Cost., 42(1), 7-30. https://doi.org/10.25268/bimc. invemar.2013.42.1.57

Wang, J., Tan, Z., Peng, J., Qiu, Q. \& Li, M. (2016). The behaviors of microplastics in the marine environment. Mar. Environ. Res., 113, 7-17. https://doi. org/10.1016/j.marenvres.2015.10.014

Weinstein, J. E., Croker, B. K. \& Gray, A. D. (2016). From macroplastic to microplastic: degradation of highdensity polyethylene, polypropylene, and polystyrene in a salt marsh habitat. Environ. Toxicol. Chem., 35(7), 16321640. https://doi.org/10.1002/etc.3432

Wright, S., Thompson, R. \& Galloway, T. (2013). The physical impacts of microplastic on marine organisms: A review. Environ. Pollut., 178, 483-492. https://doi.org/10.1016/j. envpol.2013.02.031 